\title{
Patrick Dinslage, Edvard Grieg und seine Zeit, Laaber: Laaber 2018
}

Schlagworte/Keywords: Edvard Grieg; Liedkomposition; music and biography; Musik und Biografik; Norway; Norwegen; poetic counterpoint; Poetischer Kontrapunkt; song composition

\section{I. \GROßE KOMPONISTEN UND IHRE ZEIT<}

Nicht von ungefähr verleiht Jan Brachmann in seiner Rezension derselben Publikation seiner Bestürzung darüber Ausdruck, dass »die Reihe wirklich noch so heißt«. ${ }^{1}$ In Zeiten ebenso pluralistischer wie gebrochener Perspektiven auf musikgeschichtliche Zusammenhänge, poststruktureller wie posthumaner Ansätze und einer subjektkritischen Auseinandersetzung mit rabendländischen` Prämissen ist es tatsächlich schwer vorstellbar, dass eine am `BeethovenParadigmar entwickelte Gegenüberstellung von Kunstwerk und Biographie nicht von vornherein die Annäherung an eine Musik und einen Komponisten erschwert, die schon im 19. Jahrhundert selten unvoreingenommen, sondern meist im Spannungsverhältnis divergierender nationaler und ästhetischer Konzeptionen aufgenommen wurde (7).

Patrick Dinslage entzieht sich weder einer eingehenden Auseinandersetzung mit biographischen Details noch der akribischen AnalyseArbeit. Beide von Carl Dahlhaus noch als schwer vermittelbar bezeichnete Aspekte von Biografik ${ }^{2}$ sind sogar mit besonderer Aufmerksamkeit für scheinbare 'Kleinigkeiten ‘ ausführlich behandelt, allerdings unter einem Leitgedanken, der von einigen Kerngedanken einer Kompositionsgeschichte als "Strukturgeschichte " ${ }^{3}$ oder von einer Vorstellung musikalischer Analyse als streng textbasierter "Strukturanalyse ${ }^{4}$ nicht weiter entfernt sein könnte. Dinslage stellt sowohl in der sehr durchdachten Chronik als auch im Vorwort die Interdependenz zwischen den kulturellen und politischen Umbrüchen im Skandinavien der zweiten Hälfte des 19. Jahrhunderts (8) und Griegs Biographie dar, zumal da Griegs autobiographische Deutungs-

\footnotetext{
1 Brachmann 2018, L 20.

2 Dahlhaus 1987, 29-30 und 2017, 47.

3 Dahlhaus 2017, 50.

4 Dahlhaus 1977, 6.
}

muster nicht nur auf jene Entwicklungen zurückzuführen sind, sondern sie auch nicht wenig beeinflusst haben.

\section{LEITMETAPHERN EINES GRIEG-BILDS}

Es ist mehr der Aufbau des Buches und die planvolle Auswahl biografischer Informationen, die auf eine Künstlerpersönlichkeit und ihr Umfeld schließen lassen, deren geschichtlicher Abdruck in den Bereich musik-, kunst- und sprachwissenschaftlicher, aber auch medienwissenschaftlicher und kulturpolitischer Forschungen fällt. Versucht man so etwas wie Leitmetaphern (`conceptual metaphors $\varsigma)^{5}$ des sich so zusammenfügenden Griegs-Bildes zu rekonstruieren, könnten diese mit ‘Reise` (220-221), >Dokumentation (215-225 und 226-234), 'Sprache` (154-185) und 'private Öffentlichkeit (235-273 und passim) beschrieben werden.

Dinslage arbeitet Griegs Reisewege für seine Konzerttourneen, aber auch seine Korrespondenzen und Reisen nach Europa auf, um Griegs Aktivitäten in einem kulturellen Netzwerk zwischen Skandinavien und Europa nachvollziehbar zu machen, womit gleichzeitig deutlich wird, dass so etwas wie das fast paradoxe Bild einer snordische Fernes, die dem Grieg-Bild schon damals anhaftete, so gut wie nie kulturellen, regionalen oder politischen Gegebenheiten entsprach: Es war normal (und zwar bis weit in das 20. Jahrhundert hinein), wenn skandinavische Musikerinnen und Musiker an deutschen und österreichischen Konservatorien und Hochschulen studierten, die Verkehrsverbindung zwischen Bergen und Leipzig - und zwar Post- wie Personenverkehr - war direkt und noch mehr der Kontakt zwischen dem Musikalienhandel in beiden Städten. Komponisten wie Ole Bull, Rikard Nordraak, Niels Gade, Adolf Fredrik Lindblad und vor allem Grieg selbst waren europäisch nicht nur be- 
stens vernetzt, sondern arbeiteten an Europa als kulturellem Raum nicht weniger mit als Schumann, Mendelssohn, Liszt, Brahms oder Tschaikowsky. Damit rückt so etwas wie »der nordische Ton« (109, 203-214), der nicht nur Grieg attestiert wurde, mehr und mehr in die Sphäre der neuen europäischen Mythen des 19. Jahrhunderts, deren Entstehung - wie z. B. für Deutschland die Renaissance des Kyffhäuser-Mythos ${ }^{6}$ eng mit der politischen Selbstbehauptung der s National-Staaten $<$ einherging.

Die Leitmetapher >Dokumentation`, die sich am stärksten im Kapitel über Griegs Haushaltsbücher niederschlägt, spielt in dieser Biografie auf mehreren Ebenen eine Rolle, nämlich nicht nur inhaltlich als Aspekt der Persönlichkeit Griegs, für den das Führen von Listen über das eigene Tun sowie das autobiografische Schreiben über seine Arbeit und über seine Rolle als Komponist in Norwegen und Europa von essentieller Bedeutung gewesen sein muss, sondern auch auf struktureller Ebene. Dinslage wählt im Geiste frühester historistischer Geschichtsschreibung $^{7}$ Dokumente und (Selbst)Zeugnisse aus, die er in verschiedensten Konstellationen zum Sprechen zu bringen versucht. Dabei sind Dokumentationen über Fahrkartenkäufe aus Griegs Haushaltsbüchern (222) als Zeugnisse nicht weniger swertvoll als Harmoniemodelle oder Beobachtungen in Griegs Satztechnik.

Die Beiträge über Griegs Liedkomposition sowie über sein musiktheatralisches Schaffen gewinnen vor allem dort an analytischer Tiefe, wo Dinslage, immer belegt durch Selbstzeugnisse Griegs, die Unterschiede zwischen einer historisch informierten Ästhetik norwegischer Sprache und ihrer Idiome und den jeweiligen Übersetzungen der Texte ins Deutsche oder Englische herstellt. Diese bilinguale Perspektive, die ja auch für Grieg selbst vorausgesetzt werden muss, ist nicht zuletzt deshalb von Belang, weil Grieg selbst das Verhältnis zur Sprache und ihren Ausdruckswelten für sein eigenes Komponieren sehr hoch bewertet hat (174).

Die Leitmetapher sprivate Öffentlichkeit prägt implizit die gesamte Publikation, denn zahlreich sind die teilweise paradoxen Gegen-

6 Münkler 2009, 38.

$7 \quad$ Burckhardt 2000, 777. überstellungen zwischen Edvard und Nina Griegs sehr auf private Rückzugsmöglichkeiten bedachtem Verhalten und den in jeder Hinsicht öffentlichen Konzepten, die das Ehepaar in seiner gemeinsamen Arbeit an Griegs Werk vertrat $(10,235-273)$. Am deutlichsten wird die spannungsvolle Abhängigkeit zwischen als privat markierten Details und öffentlichen bzw. intersubjektiven Diskursen in den musikalischen Analysen: So stellt Dinslage mehrfach Bezüge zwischen einer `Störung، standardisierter Prozesse, wie z. B. der Modulationsordnung in der Sonatenform, und dem wörtlichen Beharren auf personalisierten Kleinstmotiven wie den Initialen (88) E-G-H (Edvard Hagerup Grieg), der Verbindung zwischen seinen Initialen und denen seiner Frau (98) und schließlich dem sogenannten "Grieg-Motiv« (64), z. B. $c-h-g$, her, letzteres in der Analyse von Griegs früher Klaviersonate in e-Moll op. 7.

Obwohl die Auswahl der Analysebeispiele scheinbar dem pragmatischen Grundgedanken folgt, keine der im immer noch überraschend umfangreichen OEuvre vertretenen Gattungen zu vernachlässigen, markiert Dinslage eine poetische Gegenstruktur zur Gattungssystematik, indem er einen Schwerpunkt auf die Sammlung der >Lyrischen Stücke، setzt. Die von 1864 bis 1901 entstandenen Stücke werden als Kompositionswerkstatt und sozusagen als Privatissimum Griegs charakterisiert (69), die Stücke selbst dokumentieren seismographisch konzeptuelle und stilistische Entscheidungen, die auch die für eine größere Öffentlichkeit gedachten Werke kennzeichnen. Bemerkenswert ist Dinslages Deutung, die das Eröffnungsstück, die Arietta op. 12/1 und ihr finales Gegenstück, die Nachklänge op. 71/7, als autoprotokollarische Zeugnisse Griegs, als eine Art musikalisches Tagebuch mit dem Verweis auf Edvard Munchs Zyklus Lebensfries charakterisiert (69). Dabei wendet er besondere Aufmerksamkeit auf Griegs stilistisches SelbstBewusstsein, das es ihm ermöglicht, das letzte Stück präzise als in einem `Spätstilı verfasstes Echo der Arietta zu erfinden. Dasselbe hochsensible Stilempfinden, mit dem Grieg in der Altnorwegischen Romanze op. 51 mit einem hohen Grad von Erkennbarkeit Portraits seiner Zeitgenossen und Vorbilder - Schubert, Liszt, Mendelssohn, Schumann, Beethoven, Brahms, Mussorgsky - komponierte (86), konnte er of- 
fenbar auch auf seine eigene Musiksprache übertragen in einer schier paradoxen Überlagerung von Nähe und Distanz, sodass die Leserinnen und Leser dieser Analyse von op. 12/1 und op. 71/7 sich fragen dürften, wieviel Privatheit überhaupt in derlei dokumentierbaren Quellen zu finden ist, und ob es nicht, wie bei der Familie Mendelssohn ${ }^{8}$ aus heutiger Sicht vielmehr ein Bild von Privatheit war, ein Sehnsuchtsort, der ebenso als künstlerische Inspiration diente wie jedwede Vorstellung von 'Ferner.

\section{REALISMUS}

Über Griegs Liedschaffen liegt ein besonders ausführliches Selbstzeugnis des Komponisten vor, nämlich als Kommunikation mit dem Autor Henry Theophilus Finck, der ein ausführliches Kapitel über Grieg in seiner Publikation Songs and Songwriters ${ }^{9}$ verfasste (174). In dieser Publikation wird die Ibsen-Vertonung Ein Schwan op. 25/2 in den Vordergrund gestellt. Und wieder kommt, diesmal von Seiten Fincks, der nicht nur Herausgeber der Publikation, sondern auch der erste Grieg-Biograph und Herausgeber seiner Lieder in Amerika war, eine Reihe deutlich europäisch geprägter und stilisierter Vorstellungen dessen, 'was Norwegisch ist‘, in die Charakterisierung des Liedes. ${ }^{10}$ Der selbstverständliche Wunsch, so etwas wie einen ^norwegischen Ton` auf Griegs Personalstil zu projizieren, wird auch an anderer, sehr wirksamer Stelle artikuliert, nämlich im Vorwort der Liedausgabe, wo es heißt:

\footnotetext{
Like a tree with its roots in the soil of his native country Grieg absorbed the chemical qualities of the Norwegian soil without losing any of its individualities.

The folk-music of Norway is more exotic - more "foreign « to our ears - than that of the other Scandinavian countries - Sweden and Denmark. ${ }^{11}$
}

Sicher spielt es für Finck eine Rolle, dass mit Ein Schwan wie mit Peer Gynt eine Zusammenarbeit der beiden sikonischen` norwegi-

\footnotetext{
$8 \quad$ Miller 1974.

9 Finck 1900

10 Ebd., $205 f f$.

11 Finck 1908, XIII.
}

schen Künstler Grieg und Ibsen vorliegt. Mit dieser ideellen Betonung des iNorwegischen verdrängt Finck allerdings nicht nur musikalische Details, sondern vergibt auch die Chance, sich auf für die Analyse und auch für die Komposition relevantere Bezüge zur Geschichte norwegischer Kultur einzulassen.

Möglicherweise auch aus diesem Grunde holt Dinslage eine solche Analyse des Liedes nun nach (173-185), indem er als Verstehenshorizont nicht nur die mythische Vorstellung eines nordischen Tons der europäischen Romantik ansetzt, sondern vielmehr die aktuellen Probleme norwegischer Kunst und ihres Selbstverständnisses in den 1870er Jahren. Indem Dinslage die im Zuge des 'Sprachenstreits` in der Mitte des 19. Jahrhunderts parallel zur Emanzipation von Dänemark sich etablierende Unterteilung in drei Varianten des Norwegischen - die ländliche Dialektsprache 'Landsmålı, die dänisch beeinflusste Sprache der Städte ,Bokmål und schließlich das aus nationalromantischen Gründen aus alten norwegischen Dialekten künstlich entwickelte sNynorsk (158) - als für Griegs Liedvertonungen deutlich unterscheidbare Stil- und Ausdrucksträger benennt, folgt er nicht nur eigenen Aussagen Griegs, der von der "poetischen Kraft» (158) des 'Nynorskı ebenso inspiriert war wie von der »Wärme« des 'Landsmål (159), sondern zeichnet auch einen historisch angemesseneren Hintergrund derjenigen Vorstellungsräume, die ein snorwegischer Ton` für Griegs Phantasie eröffnet haben könnte. `Ferne` oder `Fremdheit spielen darin eine eher untergeordnete Rolle. In diesem durch die verschiedenen Sprachebenen nun sehr engmaschigen System für Tonfälle und Rhythmen, die im Übrigen in keiner Übersetzung zu erkennen sind (176), entsteht vielmehr ein sehr spräsentischer Bezug zur Ausdruckswelt der von Grieg ausgewählten Gedichte, ein Umgang mit Zeitwahrnehmungen, die mit Fincks Wort "Realismus « ${ }^{12}$ nur missverständlich wiedergegeben werden können. In den Analysen des in 'Landsmålı verfassten Ved Gjaetle-bekken/Am Bergbach aus dem Zyklus Haugtussa von Arne Garborg, das Grieg als op.67/8 vertonte (166-167), und dem ebenfalls in >Landsmål gedichteten Våren/Letzter Frühling op. 33/2 von Aasmund

12 Ebd. 
Olavson Vinje (158-160), aber auch des im 'Bokmål geschriebenen Der skreg en fugl./Ein Vogel schrie von Vilhelm Krag, Griegs op. 60/4, arbeitet Dinslage ein bestimmten Zügen des Impressionismus nahestehendes Stilidiom heraus, wenn es nicht um harmonische Logik, sondern vor allem »um den 'Eindruckı der Klänge in ihrer Abfolge« (164) geht, sodass sich, auch für das spätere Ein Schwan, eine vor dem Hintergrund deutscher Liedkomposition dieser Zeit neue ästhetische Umgebung entfaltet, nämlich eine sehr intensive Form von Gegenwärtigkeit oder - ungeschützt gesprochen ein Fehlen von rekonstruierter Vergangenheit oder der Dokumentation des 'Gewordenseins`, was den traditionellen Erwartungen musikalischer Formprozesse und dem Prinzip der sentwickelnden Variation sentspräche.

So ist eine der Einsichten aus Dinslages Analyse von Ein Schwan, dass die harmonische Anlage des Liedes nämlich mit bemerkenswerter Konsequenz auf einem einzigen plagalen Akkordpendel beruht, auch als Artikulation einer derart neuen Konzeption musikalischer Zeit zu verstehen. Dem wäre sicher aus der Perspektive "poetischen Kontrapunkts" - eine Kategorie, mit der Dinslage vielfach arbeitet $(137,183,209$ und passim) - noch hinzuzufügen, dass in dieses Pendel, jenseits sonatenhafter Teleologie, sowohl das `Grieg-Motiv (T. 5), aber auch das damit verbundene Tetrachord $f$-e-d-c (T. 28 bis Schluss, im Schlusstakt selbst und T. 5-6) und seine chromatischen Varianten f-e-es-d-c (T. 5-7), f-es-des-c (T. 26-28), sowie verkürzt f-des-c (z. B. T. 3-4) durchgehend präsent sind, als gelte es, einen Augenblick zu verlängern. In der Formelhaftigkeit von Singstimme und Klaviersatz, in der Dissonanzen nicht mehr für Folgelogik oder eine Vektorisierung der Taktmetrik sorgen (176), sondern stattdessen eine strenge zweitaktige Choreographie und eben diese Form unscharfer Wiederholung ähnlicher Motive formal ordnend eintritt, manifestiert sich auch im kontrapunktischen Verhalten der Stimmen ein sanderer Umgang mit musikalischer Zeit: Könnte man buchstäblich den poetischen Kontrapunkt des Liedes als eine einzige Seitenbewegung zum Liegeton $f$ bezeichnen, zu dem sich Klavierund Gesangsstimme in fast heterophoner Unabhängigkeit voneinander verhalten, so geht es hier weniger um musikalische Zeit, die sich an der Einbindung von Motiven in lineare oder diskursive Prozesse hörend erfassen lässt, sondern um das immer wieder erneute Andauern und Vergehen eines Moments. Natürlich entwickelt das Lied in der Interaktion von Harmonik und Form Hierarchien, Höhe- und Tiefpunkte $(177,178)$, aber auf der Basis eines Kontrapunkts, der sich traditionellen, zielgerichteten oder dialektischen Spannungs- und Entspannungsvorgängen zu entziehen scheint. Ein Schwan ist dem Kontrapunkt Ravels (z. B. in der Chanson épique aus Don Quichotte à Dulcinée) deutlich näher als dem ebenfalls in vieler Hinsicht vergleichbaren Schwanengesang op. 23/3 von Franz Schubert. Dass diese neue Qualität als Konsequenz einer ästhetischen Durchdringung von Naturerlebnis, (neuer) Sprache und Musik zu verstehen ist, lässt Dinslage in der Analyse von Ein Schwan auf inspirierende Weise nacherleben.

Während das Buch die aktuelle GriegLiteratur gründlich rezipiert, ist es vielleicht ein bisschen zu bedauern, dass der Anschluss an neuere Modelle der Systematisierung von romantischer Harmonik nicht gesucht wird. Den Analysen und ihrer Aussagekraft tut das keinen Abbruch, dennoch hätte vor dem disziplinären Hintergrund, dass ein Verständnis von Tonalität und Kontrapunkt aus einer rekonstruierten historischen und theoretischen Perspektive stärker von Grieg beeinflusst sein sollte, und dass sich die Auseinandersetzung mit Grieg auch in modernen systematischen Ansätzen niederschlagen sollte, ein solcher Kontakt das Buch noch wertvoller gemacht.

In der kunstvollen filmischen DerridaDokumentation von $2002{ }^{13}$ verwandelt Derrida die Schiebebewegung der différance in eine Aussage über die Biographie des Philosophen:

\footnotetext{
That's why I would say that sometimes the one who reads a text by a philosopher - for example a tiny paragraphe - and interprets it in a rigourous, inventive and powerfully deciphering fashion is more of a real biographer than the one who knows the whole story. ${ }^{14}$
}

Edvard Grieg und seine Zeit vollzieht diese hermeneutische Verschiebung zwischen "winzigen Paragraphen « - seien es nun Kleinstmoti-

Derrida 2002.

14 Ebd., 7:00-7:29. 
ve oder Fahrkartenkäufe - auf »die ganze Geschichte« in vielen Momenten. Die prekäre Vorgabe 'Werkbiographie sird damit ebenso ausgehöhlt wie behutsam modernisiert, sodass offensichtliche Probleme einer rigiden 'Struk- turanalyse ‘ wie die grundsätzliche Inkommensurabilität von Biographie und Werkbetrachtung sich nicht mehr stellen.

Ariane Jeßulat

\section{Literatur}

Brachmann, Jan (2018), „Seinen Ton erkennt man gleich «, Rezension in der FAZ vom 6.10.2018, Nr. 232, L20.

Burckhardt, Jacob (2007), Das Geschichtswerk $B d . I$ (1880), Neu Isenburg: Melzer über Zweitausendeins.

Dahlhaus, Carl (1977), Funkkolleg Musik, Studienbegleitbrief 0, Weinheim und Basel: Beltz / Mainz: Schott.

Dahlhaus, Carl (1987), Ludwig van Beethoven und seine Zeit, Laaber: Laaber.

Dahlhaus, Carl (2017), Grundlagen der Musikgeschichte (1977) mit einer kommentierenden Einführung von Michele Callela, Laa-

Eubanks, Philipp (1999), »The Story of Conceptual Metaphor: What Motivates Metaphoric Mappings", Poetics Today 20/3, 383-396.

Finck, Henry Theophilus (1900), Songs and Song Writers, New York: C. Scribner's Sons.

Finck, Henry Theophilus (1908), Fifty Songs by Edvard Grieg, Philadelphia: J. E. Ditson \& Co.

Miller, Norbert, »Felix Mendelssohn Bartholdys italienische Reise«, in: Das Problem Mendelssohn, hg. von Carl Dahlhaus, Regensburg: Bosse, 23-34.

Münkler, Herfried (2009), Die Deutschen und ihre Mythen, Berlin: rowohlt.

Derrida, Jacques (2002), Derrida. Ein Film von Kirby Dick und Amy Ziering Kofman, Jane Doe Films/Suhrkamp Realfiction.

Jeßulat, Ariane (2019): Patrick Dinslage, Edvard Grieg und seine Zeit,

Laaber: Laaber 2018. ZGMTH 16/1, 153-157.

https://doi.org/10.31751/1006

(C) 2019 Ariane Jeßulat (ajessulat@aol.com)

Universität der Künste Berlin

Dieser Text erscheint im Open Access und ist lizenziert unter einer

Creative Commons Namensnennung 4.0 International Lizenz.

This is an open access article licensed under a

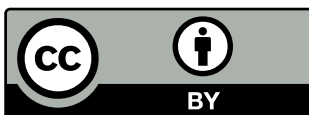

Creative Commons Attribution 4.0 International License.

eingereicht / submitted: 19/05/2019

angenommen / accepted: 21/06/2019

veröffentlicht / first published: 30/06/2019

zuletzt geändert / last updated: 30/06/2019 